\title{
Correlação entre os estágios de mineralização dentária na arcada inferior e a idade esquelética. Revisão da literatura
}

\author{
Luiz Fernando Eto*, Enio Tonani Mazzieiro**
}

\begin{abstract}
Resumo
O objetivo do presente trabalho foi o de revisar a literatura buscando uma correlação entre os estágios de mineralização dentária e a idade esquelética. Enquanto alguns trabalhos não encontraram nenhuma correlação entre essas idades biológicas, outros mostraram uma íntima relação entre elas, sugerindo a determinação da idade esquelética a partir da mineralização radicular de dentes específicos, principalmente no arco inferior. Portanto, um levantamento minucioso das opiniões acerca do assunto poderá colaborar para uma melhor compreensão das controvérsias existentes.
\end{abstract}

Palavras-chave: Mineralização dentária. Surto de crescimento puberal. Idade esquelética.

\section{INTRODUÇÃO}

Para alcançar a maturidade, um indivíduo passa por processos contínuos de desenvolvimento. Segundo Marcondes ${ }^{30}$, a maturidade é o produto final de um processo, isto é, um estado acabado e a maturação é o processo através do qual este estado é atingido.

O desenvolvimento do homem possui características próprias, com dois períodos de aceleração ao longo do crescimento. O primeiro desses dois períodos ocorre entre as idades de seis a oito anos e é chamado de surto de crescimento infantil. O segundo, mais acentuado e evidente, ocorre durante a puberdade, envolvendo os fenômenos físicos que acompanham a maturação do aparelho genital e o alcance da capacidade reprodutiva ${ }^{33}$, sendo chamado de surto de crescimento puberal (SCP). Para a Ortodontia, o SCP apresenta grande importância, pois nessa época, melhores resultados de tratamento são obtidos num período de tempo relativamente curto, principalmente na presença de más oclusões associadas a desvios esqueléticos. Isto se deve à relação existente entre o $\mathrm{SCP}$ e as dimensões faciais da maxila e da mandíbula ${ }^{4}$. De acordo com Chertkow $^{10}$, quando se possui uma informação precisa desse surto, pode-se determinar se o pico de velocidade de crescimento é eminente, presente ou completo.

Para determinar o estágio que um indivíduo atingiu no seu desenvolvimento, podemos utilizar, além da idade cronológica, a idade fisiológica e a idade biológica ${ }^{44}$. As três formas mais utilizadas

\footnotetext{
* Especialista e Mestre em Ortodontia pela PUC-MINAS. Professor do curso de especialização em Ortodontia da F.O.U. Itaúna.

** Doutor em Ortodontia pela USP-Bauru, Profesor Adjunto III da PUC/Minas, Coordenador do Mestrado em Ortodontia da

PUC/Minas.
} 
para a determinação da idade biológica são o grau de desenvolvimento do esqueleto, do sistema genital e dos dentes.

A idade esquelética é melhor determinada por radiografias de mão e punho. A detecção do aparecimento de ossos específicos no carpo e metacarpo e a observação da união epifisária das falanges podem definir o grau de maturação esquelética do paciente. Bowden ${ }^{5}$, Brown $^{7}$, Fishman ${ }^{16}$, Grave, Brown ${ }^{19}$, Hägg e Taranger ${ }^{22}$, Hunter $^{25}$ e Tanner ${ }^{42}$ são alguns dos pesquisadores que ajudaram a consagrar na literatura este tipo de avaliação como o mais preciso e sensível método para determinação da idade esquelética. A partir destes dados, eventos específicos de mineralização, que ocorrem em uma seqüência progressiva, passaram a ser correlacionados com o crescimento puberal ${ }^{6,16,19,23,32,33}$. Esse fato permitiu se traçar um gráfico do surto de crescimento, possibilitando uma visualização simples e objetiva dos estágios de maturação dos pacientes ortodônticos e a definição da melhor época para as intervenções ortopédicas.

A análise da idade do sistema genital e da idade dentária mostrou sofrer maiores variações do que a idade esquelética na avaliação do grau de maturidade fisiológica de uma criança em crescimento $^{13,20,27,42}$.

A associação entre a idade esquelética e a idade dentária tem-se mostrado inconsistente em alguns trabalhos. Os estudos comparativos entre a idade dentária, o número de dentes presentes na cavidade bucal e o grau de mineralização de vários dentes, normalmente mostram pouca ou nenhuma correlação entre estes e outros indicadores de maturação $0^{1,3,37}$. Entretanto, alguns pesquisadores ${ }^{10,11,14,15,18,36,38,40}$ têm encontrado uma estreita relação entre os estágios de mineralização dentária e o início do surto de crescimento puberal. Dos dentes pesquisados, os que têm demonstrado uma menor variabilidade e conseqüentemente uma alta correlação com a idade esquelética têm sido os caninos inferiores ${ }^{10,11,40}$ e, recentemente, tam- bém os segundos molares inferiores ${ }^{36,38}$.

Uma análise pormenorizada dos trabalhos que relacionam os estágios de mineralização radicular e o SCP mostra que apesar das evidências apresentadas, ainda existem muitas dúvidas e controvérsias acerca do assunto. Para citar algumas, o trabalho de Chertkow ${ }^{10}$ não encontrou relação entre as fases de mineralização dos segundos molares inferiores no gênero feminino e o SCP, sendo este o grande resultado do recente trabalho de Rossi, Amorin e Pacheco ${ }^{38}$.

A relação do segundo molar inferior com o SCP no trabalho de Moraes, Médici Filho e Moraes ${ }^{36}$ não levou em consideração o dimorfismo sexual e entretanto foi encontrado o mesmo resultado do trabalho de Rossi, Amorim e Pacheco ${ }^{38}$ que só utilizaram amostra do gênero feminino. Chertkow ${ }^{10}$ afirmou que, com exceção dos caninos inferiores, existem diferenças nos estágios de mineralização dos dentes entre indivíduos de gêneros distintos assim como, de maneira geral, em indivíduos de diferentes etnias.

Portanto, o objetivo deste trabalho é revisar a literatura procurando entender o motivo das controvérsias existentes. Caso se confirme a hipótese da possibilidade de se prever o SCP por meio de radiografias dentárias, o ortodontista passaria a possuir mais um subsídio para predizer a época do SCP de um indivíduo. Como as radiografias dentárias (panorâmicas ou periapicais) são documentos de rotina necessários ao diagnóstico ortodôntico, elas estariam colaborando na informação da predição do momento de crescimento dos pacientes, sem a necessidade de se estar tomando radiografia de mão e punho. Isto iria ao encontro dos anseios de muitos profissionais, dos quais citamos Santos e Almeida ${ }^{39}$, segundo os quais, atualmente existe uma grande preocupação em se reduzir as exposições radiográficas aos pacientes.

\section{REVISÃO DE LITERATURA}

Uma vez determinada a idade esquelética de um indivíduo, pode-se posicioná-lo na curva de 
crescimento puberal pela seqüência de eventos que ocorrem na mão e punho ${ }^{22,31,33}$. Entretanto, pouco se sabe da relação entre o surto de crescimento puberal e a idade dentária. Como visto anteriormente, vários são os autores que afirmaram que a idade esquelética é a mais precisa para se determinar o SCP. Apesar disso, existem pesquisadores que encontram uma grande correlação entre a idade dentária e o SCP, observando-se as fases de mineralização dos caninos inferiores ${ }^{9}, 10,11$, dos segundos molares inferiores ${ }^{36,38}$ e dos terceiros molares inferiores ${ }^{15}$ com a idade esquelética, sugerindo-se assim, uma associação das fases de mineralização dentária como indicadoras da adolescência.

Lamons e $\mathrm{Gray}^{27}$ estudaram a relação entre a idade de erupção dos dentes, a idade esquelética e a idade cronológica em 61 indivíduos americanos, com a finalidade de saber se existia crescimento semelhante em partes diferentes de um mesmo corpo. Os resultados obtidos mostraram que a idade cronológica é um índice um pouco melhor para se determinar o desenvolvimento dos dentes do que a idade esquelética. Eles ressaltaram, entretanto, que foram utilizadas muitas medidas de uma mesma criança em diferentes épocas, o que pode ter comprometido os resultados obtidos. Os autores sugerem mais trabalhos a respeito, uma vez que esse foi um dos primeiros a tratar deste assunto.

Lewis e Garn ${ }^{29}$ tentaram obter a relação entre a formação dentária e outros fatores de maturação. Utilizaram 255 crianças americanas e obtiveram radiografias oblíquas da mandíbula além de uma grande quantidade de informações sobre o crescimento somático, sexual, e dados sobre o comportamento, a personalidade e a saúde em geral. Os dentes posteriores inferiores foram analisados com relação aos seus estágios de mineralização e estágios de emergência na cavidade bucal. A idade esquelética foi analisada através da avaliação de radiografias carpais. Como conclusão de seus estudos eles afirmaram que o desenvolvimento dentário não é modificado pelas variações do crescimento; observaram uma grande variabilidade durante os períodos de mineralização dentária e que a relação entre a formação dos dentes e a maturação geral necessita de estudos mais aprofundados.

Um dos primeiros autores que procurou investigar e correlacionar os valores de vários índices físicos de desenvolvimento foi Green ${ }^{20}$. Ele concluiu que a idade dentária mostrou um alto grau de correlação com a idade cronológica $(0,6774)$, e baixa correlação com a idade esquelética $(0,4616)$. Altura e peso mostraram um alto grau de correlação $(0,8145)$. Entretanto, o autor sugere estudos mais aprofundados nos indicadores esqueléticos, além de uma avaliação mais detalhada da idade dentária, uma vez que foram utilizados somente os dentes inferiores posteriores em seu trabalho.

Garn, Lewis e Bonné ${ }^{18}$ avaliaram o desenvolvimento dos terceiros molares inferiores, incluindo a possibilidade de correlação com a maturação esquelética em 140 indivíduos americanos. Concluíram dizendo que foi encontrado um alto grau de variabilidade na mineralização dos terceiros molares para os nove estágios observados e que a interrelação da idade de formação desses dentes com a maturação esquelética foi baixa e não significante.

Anderson, Thompson e Popovich ${ }^{2}$ utilizaram 121 adolescentes do gênero masculino e 111 adolescentes do sexo feminino do grupo experimental do "Burlington Growth Center", E.U.A, para avaliar a correlação entre os estágios de mineralização dentária, mineralização esquelética e altura e peso do corpo. Os estágios de mineralização dos dentes de um lado da maxila e mandibula foram analisados de acordo com o método de Moorrees, Fanning e Hunt $\mathrm{Jr}^{3}{ }^{34}$. Um total de 13 ossos da mão e do punho direito foram analisados de acordo com o método de Greulich e Pyle ${ }^{21}$. Altura e peso foram obtidos a partir de registros anuais. Eles concluíram que a idade dentária poderia ser relacionada às idades esqueléticas, peso e altura. O desenvolvimento dos dentes esteve mais relacionado com 
o desenvolvimento morfológico do que com o desenvolvimento esquelético em ambos os gêneros. Os autores chamam atenção para o fato de que, para investigações que envolvam idade dentária, é preferivel considerar a idade de um dente específico do que expressá-la pela idade média de um grupo de dentes.

Chertkow ${ }^{10}$ foi um dos primeiros a encontrar uma alta correlação entre os estágios de mineralização dos caninos inferiores com os eventos de mineralização. Para isto, ele utilizou 197 pacientes de ambos os gêneros, sendo 159 sul-africanos leucodermas e 38 melanodermas. Foram utilizadas radiografias panorâmicas ou laterais oblíquas da mandíbula e maxila juntamente com as radiografias de mão e punho. Após a utilização do teste qui-quadrado, a maior contribuição veio dos estágios $\mathrm{F}$ e $\mathrm{G}$ dos caninos inferiores, que mostraram uma grande correlação com a mineralização do gancho do hamato e com o capeamento da falange média do terceiro dedo. Ele concluiu seu trabalho afirmando que o estudo da formação radicular do canino inferior pode ser usado clinicamente como um indicador do surto de crescimento puberal em um sul-africano de origem caucasiana. Pede atenção, entretanto, ao tentar extrapolar esta correlação para indivíduos de outros grupos raciais.

C. Engstrom, H. Engstrom e Sagne ${ }^{15}$ investigaram o desenvolvimento dos terceiros molares em relação à idade cronológica e esquelética. Como conclusão de seus trabalhos, os autores encontraram uma forte correlação entre a idade cronológica e o desenvolvimento dos terceiros molares em ambos os gêneros, entre a idade cronológica e a maturação esquelética e entre o desenvolvimento do terceiro molar e a maturação esquelética $(\mathrm{r}=0.77)$. Eles discutem seus resultados sugerindo que um número menor de estágios de mineralização associado a uma mineralização mais longa do terceiro molar permite uma comparação mais precisa com os eventos de maturação esquelética.

Demirjian et al. ${ }^{13}$ avaliaram a interrelação entre as idades de maturidade esquelética, somática, sexual e dentária de 50 indivíduos canadenses do gênero feminino, com idade variando entre seis e 15 anos. Os autores concluíram que a maturidade esquelética, somática e sexual são interrelacionadas presumivelmente por um mecanismo de controle comum. O desenvolvimento dentário não está relacionado com estes índices de maturação e parece ser controlado por outros sistemas de desenvolvimento.

Sierra ${ }^{40}$ avaliou a correlação existente entre os estágios de desenvolvimento de centros específicos de crescimento da mão e punho e a mineralização dos caninos superiores e inferiores, pré-molares e segundos molares. Ela justificou seu trabalho em função dos resultados conflitantes até então expostos na literatura. Segundo a autora, em caso de uma correlação entre os dois fenômenos, o nível de mineralização dos dentes permanentes poderia ser utilizado efetivamente para a estimativa do nível de desenvolvimento esquelético dos pacientes. Sua amostra consistiu de 153 crianças caucasianas de oito a 12 anos de idade tratadas no Departamento de Ortodontia da Universidade de Detroit, E.U.A. Ela conclui seu trabalho afirmando que a estreita correlação entre a maturação esquelética e a idade dentária encontrada em seu trabalho sugere que a determinação radiográfica da maturação esquelética é de menor importância clínica no tratamento de crianças que apresentam um padrão normal de desenvolvimento, principalmente através da avaliação dos estágios de mineralização dos caninos inferiores.

Gandini et al. ${ }^{17}$ procuraram obter uma correlação entre a idade esquelética e dentária sugerindo utilizar somente uma radiografia panorâmica na determinação da idade dentária e do pico de crescimento puberal em uma tentativa de simplificar o procedimento de diagnóstico, além de diminuir a exposição radiográfica. Foram utilizadas 116 radiografias panorâmicas e de mão e punho de crianças italianas de oito a 15 anos de idade. Os resultados do autor mostram ser impossível estimar a maturação esquelética através da análise 
dentária somente.

Lewis $^{28}$ publicou um trabalho sobre comparações entre idades dentária e esquelética. Ele concluiu afirmando que, em algumas crianças, a diferença entre as idades chegou a 36 meses; a diferença foi menor do que seis meses em $40 \%$ das crianças; a análise de regressão mostrou uma associação não mais do que moderada entre as duas idades, pois foram obtidas variações de até um ano entre elas; o baixo nível de associação provavelmente reflete o fato de que maturação dentária e maturação esquelética são processos distintos e portanto nenhuma destas idades pode ser prevista, com precisão a partir da outra.

Hypólito Silva ${ }^{24}$ utilizou o grau de mineralização do segundo pré-molar inferior para avaliar a possibilidade de se determinar a idade esquelética de pacientes. Foram utilizadas radiografias panorâmicas e de mão e punho de 33 pacientes. A autora concluiu que o grau de mineralização do segundo pré-molar inferior não pode ser usado como indicador clínico da ocorrência do surto de crescimento puberal.

Um trabalho que avaliava a relação entre os estágios de mineralização especificamente dos caninos inferiores com a maturidade esquelética foi elaborado por Coutinho, Buschang e Mirnada ${ }^{11}$. Eles utilizaram 200 adolescentes do gênero masculino e 215 do gênero feminino que procuravam por tratamento ortodôntico. A idade esquelética foi determinada de acordo com os métodos descritos por Greulich e Pyle ${ }^{21}$. Os estágios de maturação das falanges e o surgimento do adutor sesamóide foram definidos pelo método de Tanner e Whitehouse ${ }^{41}$. O desenvolvimento do canino inferior foi medido de acordo com os estágios de mineralização dentária de Demirjian, Goldstein e Tanner ${ }^{12}$. Os resultados do trabalho indicaram, segundo os autores, que a mineralização dos caninos inferiores pode servir como uma ferramenta útil para a avaliação da maturação esquelética dos adolescentes e, por associação, à maturação somática.
Kullman ${ }^{26}$ testou a precisão de dois métodos de avaliação da idade dentária por ele proposto, para avaliar terceiros molares de adolescentes suecos e tentaram obter uma interrelação entre a idade dentária, idade esquelética e idade cronológica desses indivíduos. Foram utilizados 72 indivíduos suecos, de ambos os gêneros de 13 a 24 anos de idade que haviam se submetido a tratamento ortodôntico. $\mathrm{O}$ autor concluiu que não houve uma correlação entre a idade esquelética e a idade dentária dos terceiros molares, uma vez que esses dentes sofrem uma gama muito extensa de variações durante seu processo de desenvolvimento.

Um trabalho que procurou verificar a importância do fator genético sobre o padrão de maturação de distintas estruturas foi o de Vallejo-Bolaños e España-Lopes ${ }^{43}$. Eles fizeram uma correlação entre idade dentária, esquelética e cronológica de 54 crianças originadas de famílias de baixa estatura. Essas crianças possuíam o desenvolvimento genital e a concentração de hormônio de crescimento normais, além de não possuírem nenhuma desordem patológica. A taxa de crescimento dessas crianças era sempre abaixo do normal, porém uniforme. Elas pareciam completamente normais exceto pela sua estatura. Os resultados mostraram que a idade dentária estava atrasada em relação à idade cronológica. A idade esquelética estava atrasada em relação à idade dentária e à idade cronológica. A idade dentária esteve mais relacionada com a idade cronológica do que com a idade esquelética, porém todas as correlações foram altas e estatisticamente significantes.

Outro trabalho que utilizou indivíduos de baixa estatura e comparou seu crescimento craniofacial e idade dentária foi o desenvolvido por Van Erum et al. ${ }^{45}$. Foram utilizados 48 indivíduos com baixa estatura e por isso submetidos a tratamento com altas dosagens de hormônio de crescimento. Os objetivos eram obter informações a respeito da maturação e do crescimento craniofacial, além de avaliar os possíveis efeitos das altas doses do hormônio de crescimento nesses indivíduos. 
Os resultados mostraram que o atraso no crescimento geral se reflete no complexo craniofacial. A maturação dentária parece ser um processo distinto e levemente relacionada com a idade cronológica, e independente do crescimento geral e idade esquelética.

Em sua tese de doutorado, Moraes ${ }^{35}$ estudou por meio de radiografias panorâmicas e carpais, qual a relação entre as fases da curva padrão do surto de crescimento puberal (SCP) com as fases de mineralização dentária, idade cronológica, idade dentária e idade esquelética. A amostra utilizada constou de 244 pacientes, sendo 112 do gênero masculino e 132 do gênero feminino, com as idades cronológicas variando de 84 a 191 meses. Como resultado de seus trabalhos a autora observou, considerando os dois gêneros, que quanto à mineralização dentária, as fases mais marcantes em relação ao SCP apontam, no início da curva de crescimento, que o segundo molar está iniciando a formação radicular; no pico do SCP que o canino está completando o fechamento apical e no fim do SCP que o canino e os pré-molares estão com os ápices fechados, mas o segundo molar está terminando a formação radicular, ainda com o ápice aberto, sempre com um atraso para o gênero feminino. O fechamento apical dos caninos, pré-molares e segundos molares é um forte indicador de que o SCP já terminou. As curvas de crescimento elaboradas mostram que a mineralização dentária acompanha as fases do SCP, indicando que a relação entre elas é válida, e, desse modo, a radiografia dentária pode ser utilizada clinicamente para verificar a época do SCP.

Carvalho ${ }^{8}$ propôs avaliar a relação existente entre o desenvolvimento esquelético e dentário, utilizando radiografias de 151 crianças na faixa etária de oito anos e seis meses a 14 anos e seis meses. A autora utilizou, para uma avaliação mais precisa da idade dentária, medidas da altura radicular e largura apical dos dentes estudados. Obteve correlações negativas entre o desenvolvimento esquelético e as medidas de largura apical dos dentes avaliados em ambos os gêneros.

Em um recente trabalho sobre este assunto Rossi, Amorim e Pacheco ${ }^{38}$ correlacionam os estágios de mineralização dos segundos molares inferiores e a estimativa da maturação esquelética. Este trabalho avaliou especificamente os estágios de mineralização dos segundos molares inferiores e a mineralização da região metacarpo-falangeana do primeiro dedo, principalmente o osso sesamóide e a epífise da falange proximal. Foram feitas radiografias do dedo polegar e do segundo molar inferior, ambas do lado esquerdo. Foi verificada, como resultado do trabalho, a existência de correlação entre os estágios iniciais de mineralização radicular dos segundos molares inferiores e o início do SCP; entre o estágio intermediário de formação radicular dos segundos molares inferiores e o pico de velocidade máxima do SCP; e entre os estágios finais de mineralização radicular e o final do surto.

\section{DISCUSSÃO}

Baseado na revisão de literatura, pode-se observar que alguns trabalhos encontraram pouca ou nenhuma correlação entre as idades esquelética e dentária ${ }^{13,17,20,28,45}$, outros mostraram uma íntima relação entre elas, sugerindo a determinação da idade esquelética a partir da mineralização radicular de dentes específicos, principalmente no arco inferior. Dos dentes pesquisados, os que têm demonstrado uma menor variabilidade e conseqüentemente uma alta correlação com a idade esquelética têm sido os caninos inferiores ${ }^{10,11,40} \mathrm{e}$, recentemente, também os segundos molares inferiores $^{36,38}$.

Quando a idade dentária foi relacionada à idade esquelética, levando em consideração os terceiros molares, alguns autores ${ }^{18,26}$ concluíram que não houve correlação significante; justificando estes achados através das diversas alterações sofridas por estes dentes durante o processo de desenvolvimento. Entretanto, Engstrom, Engstrom e Sagne $^{15}$ investigaram o desenvolvimento dos ter- 
ceiros molares em relação à idade cronológica e esquelética. Como conclusão de seus trabalhos, os autores encontraram uma forte correlação entre o desenvolvimento do terceiro molar e a maturação esquelética $(r=0.77)$. Eles discutem seus resultados sugerindo que um número menor de estágios de mineralização associado a uma mineralização mais longa do terceiro molar permite uma comparação mais precisa com os eventos de maturação esquelética.

Parece que independente do dente examinado, a idade dentária está mais relacionada à idade cronológica do que à idade esquelética ${ }^{20,43}$.

\section{CONCLUSÕES}

Pelo exposto acima pode-se concluir que muitas dúvidas ainda persistem na literatura sobre a possibilidade de correlação entre as idades esquelética e dentária. Os trabalhos publicados mos- tram contradições em seus resultados e ao longo do tempo percebemos alternância de opiniões entre os autores.

As contradições dos resultados podem estar relacionadas ao tamanho das amostras utilizadas, metodologias de avaliação e validade dos resultados estatísticos. Isto deixa margem para um trabalho com metodologia clara e objetiva, amostra compativel e principalmente análise estatística adequada para os objetivos propostos.

Alem disso, resaltamos a necessidade de se avaliar tais dados em relação à população brasileira, uma vez que são extremamente escassas pesquisas direcionadas a nossa população, indiscutivelmente diferente das demais pesquisadas em relação às características genéticas, ambientais e até mesmo nutricionais.

Enviado em: Maio de 2003 Revisado e aceito: Outubro de 2003

\title{
Correlationship between the stages of tooth mineralization on the lower arch and the skeletal age. Literature review
}

\begin{abstract}
The aim of this work was to get a correlationship between the stages of tooth mineralization and the skeletal age. This work was carried on in order to clean the controverses find in the literature about this subject. While some articles don't get any correlationship between those biological age, some show a great relation between them, suggesting the possibility of determine the skeletal age using the stages of tooth mineralization.
\end{abstract}

Key words: Tooth mineralization. Puberal growth spurts. Skeletal age.

\section{REFERÊNCIAS}

1. ACHESON, R. M. The Oxford method of assessing skeletal maturity. Clin Orthop, [S.I.], v. 10, p.19-39, 1957

2. ANDERSON, D.L.; THOMPSON, G.W.; POPOVICH, F. Interrelationships of dental maturity, skeletal maturity, height and weight from age 4 to 14 years. Growth, Philadelphia, v. 39, p. 453-462, 1975.
3. BJÖRK, A. Timing of interceptive orthodontic measures based on stages of maturation. Trans Eur Orthod Soc, London, p. 61$74,1972$.

4. BJÖRK, A.; HELM, S. Prediction of the age of maximum puberal growth in body height. Angle Orthod, Appleton, v. 37, no. 2, p.134-143, Apr. 1967. 
5. BOWDEN, B. D. Sesamoid bone appearance as an indicator of adolescence. Aust Orthod J, Brisbane, v. 2, no. 6, p. 242-248, June 1971.

6. BOWDEN, B. D. Epiphysial changes in the hand/wrist area as indicators of adolescent stage. Aust Orthod J, Brisbane, v. 4 no. 3, p. 87-104, 1976

7. BROWN, T. Skeletal maturity and facial growth assessment. Aust Orthod J, Brisbane,v. 2, no. 3, p. 80-87, Feb. 1970

8. CARVALHO, D. A. Estudo da relação entre dois estimadores de maturidade: desenvolvimento esquelético e dentário. 1999. Dissertação (Mestrado) - Faculdade de Odontologia de Araraquara, Universidade Estadual Paulista, Araraquara, 1999.

9. CHERTKOW, S.; FATTI, P. The relationship between tooth mineralization and early radiographic evidence of the ulnar sesamoid. Angle Orthod, Appleton, v. 9, no. 4, p. 282-288, Oct. 1979

10. CHERTKOW, S. Tooth mineralization as an indicator of the pubertal growth spurt. Am J Orthod, St. Louis, v. 77, no.1, p. 79-91, Jan. 1980

11. COUTINHO, S.; BUSCHANG, P. H.; MIRANDA, F. Relationships between Mandibular canine calcification stages and skeletal maturity. Am J Orthod Dentofacial Orthop, St. Louis, v. 104 p. 262-268, Sept. 1993

12. DEMIRJIAN, A.; GOLDSTEIN, H.; TANNER, J. M. A new system of dental age assessment. Hum Biol, Detroit, v. 45, no. 2 p. 211-227, May 1973.

13. DEMIRJIAN, A. et al. Interrelationships among measures of somatic, skeletal, dental, and sexual maturity. Am J Orthod St.Louis, v. 88, no. 5, p. 433-438, Nov. 1985

14. DEMISH, A. E.; WARTMANN, P. Calcification of the mandibula third molar and its relation to skeletal and chronological age in children. Child Dev, Lafayette, v. 27, p. 459-473, 1956.

15. ENGSTROM, C.; ENGSTROM, H.; SAGNE, S. Lower third development in relation to skeletal maturity and chronological age. Angle Orthod, Appleton, v. 42, p. 98-105, 1983.

16. FISHMAN, L. S. Radiographic evaluation of skeletal maturation. A clinically oriented method based on hand-wrist films. Angle Orthod, Appleton, v. 52, no. 2, p. 88-111, Apr. 1982

17. GANDINI, P. et al. Dental age and skeletal age: correlation study. Mondo Ortod, Milano, v. 14, no. 2, p. 207-210, Mar./Apr. 1989.

18. GARN, S.M.; LEWIS, A. B. BONNE, B. Third molar formation and its developmental course. Angle Orthod, Appleton, v. 32, no. 4, p. 270-278. Oct. 1962

19. GRAVE, K.. C.; BROWN, T. Skeletal ossification and the adolescent growth spurt. Am J Orthod, St.Louis, v. 69, no. 6, p. 611-619, June 1976.

20. GREEN, L. J. The interrelationships among height, weight and chronological, dental and skeletal ages. Angle Orthod, Appleton, v. 31, no. 3, p.189-193, July 1961.

21. GREULICH, W. W.; PYLE, I. S. Radiographic atlas of the skeletal develepment of the hand and wrist. 2 nd ed. Stanford: Stanford Universit Press, 1959

22. HÄGG, U.; TARANGER, J. Menarche and voice change as indicators of the pubertal growth spurt. Acta Odont Scand, Stockholm, v. 38, p.179-186, 1980

23. HELM, S. et al. Skeletal maturation of the hand in relation to maximum puberal growth in body height. Tidsskr Prakt Tandlaeg. Copenhagen, v. 6, p.1223-1234, 1971.

24. HYPÓLITO, S. G. C. Avaliação do surto de crescimento puberal através do exame clínico e radiográfico dos estágios de calcificação do segundo pré-molar inferior. 1992. Dissertação (Mestrado) - Faculdade de Odontologia, Universidade Federal do Rio de Janeiro, Rio de Janeiro, 1992

25. HUNTER, C. J. The correlation of facial growth with body height and skeletal maturation at adolescence. Angle Orthod Appleton, v. 36, no.1, p. 44-54, Jan. 1966

26. KULLMAN, L. Accuracy of two dental and one skeletal age estimation method in Swedish adolescents. Forensic Sci Int Lausanne, v. 75, no. 2/3, p. 225-36. Oct. 1995

27. LAMONS, F. F.; GRAY, S. W. A study of the relationship between tooth eruption age, skeletal development age, and chronological age in sixty-one Atlanta children. Am J Orthod, St.Louis, v. 44, no. 9, p. 687-691, Sept. 1958.
28. LEWIS, A. B. Comparisons between dental and skeletal ages. Angle Orthod, Appleton, v. 61, p. 87-92, 1991.

29. LEWIS, A. B.; GARN, S. M. The relationship between tooth formation and other maturational factors. Angle Orthod Appleton, v. 30, no. 2, p. 70-77, 1960

30. MARCONDES, E. Idade óssea em pediatria. Pediatria, São Paulo, v. 2, p. 297-311, 1980

31. MARTINS, J.C.R. Surto de crescimento puberal e maturação óssea em ortodontia. 1979. Dissertação (Mestrado) - Faculdade de Odontologia, Universidade de São Paulo, São Paulo, 1979.

32. MARTINS, J. C. R.; SAKIMA, T. Considerações sobre a previsão do surto de crescimento puberal. Ortodontia, São Paulo, v.10, n. 3, p.164-170, dez. 1977.

33. MERCADANTE, M. M. N. Radiografia de mão e punho. In: FERREIRA, F. V. Ortodontia: diagnóstico e planejamento clínico. São Paulo: Artes Médicas, 1996. p.187-216.

34. MOORREES, C. F. A. FANNING, E A. HUNT Jr., E. E. Age Variation of formation stages for ten permanent teeth. J Dent Res, Chicago, v. 42, no. 6, p.1490-1502, 1963.

35. MORAES, M. E. L. Surto de crescimento puberal: relação entre mineralização dentária, idade cronológica, idade dentária e idade óssea. Método radiográfico. 1997. Tese (Doutorado em Radiologia) - Faculdade de Odontologia, UNESP, São José dos Campos, 1997.

36. MORAES, M. E. L.; MÉDICI FILHO, E.; MORAES, L. C. Surto de crescimento puberal-Relação entre mineralização dentária, idade cronológica, idade dentária e idade óssea: método radiográfico. Rev Odontol UNESP, São José dos Campos, v. 27, n. 1, p. 111-29, jan./ jun. 1998.

37. ROBINOW, M. Appearance of ossification centers. Am J Dis Child, Chicago, v. 64, p. 229-236, 1942

38. ROSSI, R. R.; AMORIM, S. G.; PACHECO, M. C.T. Correlação entre estágios de mineralização dos dentes e estimativa da maturação esquelética. Ortodontia, São Paulo, v. 32, n. 3, p. 49-57, set./dez. 1999.

39. SANTOS, S. C. B. N.; ALMEIDA, R. R. Estudo comparativo de dois métodos de avaliação da idade esquelética utilizando telerradiografias em norma lateral e radiografias carpais. Ortodontia, São Paulo, v. 32, n. 2, p. 33-45, maio/ago. 1999.

40. SIERRA, A. M. Assessment of dental and skeletal maturity. A new approach. Angle Orthod, Appleton, v. 57, no. 3, p. 194-208, July 1987.

41. TANNER, J. M.; WHITEHOUSE, R. H. Standards for skeletal age. Paris: International Children's Center, 1959.

42. TANNER, J. M. Growth at adolescence. 1st ed. Oxford: Blackwell Scientific, 1962

43. VALLEJO-BOLANÕS, E.; ESPAÑA-LOPEZ, A. J. The relationship between dental age, bone age and chronological age in 54 children with short familial stature. Int J Paediatr Dent, Oxford, v. 7, p.15-17. 1997

44. LINDEN, F. P. G. M. van Der Crescimento físico geral. In:___Crescimento e ortopedia facial.São Paulo: Ed. Santos, 1990. p.17-39.

45. VAN ERUM, R. et al. Short stature of prenatal origin: craniofacial growth and dental maturation. Eur J Orthod, London, v. 20 no. 4, p. 417-25, Aug. 1998.

\section{Endereço para correspondência}

Luiz Fernando Eto

Rua Professor Saul Macedo 212 - Belvedere

Belo Horizonte - MG

CEP: $30320-490$

E-mail: ortoeto@globo.com 